\title{
Sur quelques Haemapbysalis paléarctiques (Acariens, Ixodoidea)
}

\author{
Par P.-C. MOREL
}

DERMACENTOR PARVUS Neumann, 1897, p. 378-379.

Voici les détails les plus caractéristiques selon la description originale:

《Mâle. Corps ovale, plus large en arrière, long de 2,6 mm, large de 1,6 mm. Ecusson convexe, brun noirâtre, comme verni, glabre ;... sillons cervicaux très courts; sillon latéral peu profond, s'arrêtant au feston marginal extrême; festons à séparations bien marquées ; ponctuations très nombreuses, fines; yeux pâles situés au niveaux de la deuxième paire de pattes. Face ventrale brun rougeâtre, glabre, sillons sexuels écartés, divergents. Rostre à angles postérieurs de la base prolongés en pointes saillantes et mousses. Hypostome à nombreux denticules antérieurs, à quatre files de dents sur chaque moitié. Palpes courts, renflés en dehors, dépassant beaucoup le bord externe de la base du rostre, pourvus de poils à leur bord externe. Pattes assez fortes, brun rougeâtre. Hanches glabres, croissant peu de la première à la quatrième ; celles des deux premières paires allongées en une épine peu saillante ; une tubérosité au bord postérieur des autres... D'après un individu, probablement jeune, originaire de Syrie. »

En 1911, Neumann répète en les résumant ces données, ainsi que Arthur (1960).

On peut remarquer que l'espèce est de petite taille, sans émail (comme il peut arriver chez les individus nains des espèces émaillées ou sitôt après la mue, ce que semble vouloir exprimer Neumann par : probablement jeune). On rencontre par la suite deux caractères en désaccord avec la morphologie des Dermacentor paléarctiques. En premier lieu la hanche I n'est pas bifide (ce qui ne se rencontre que chez les Ixodes, les Haemaphysalis et les Boophilus). Il ne s'agit pas d'un Ixodes, Neumann le classe dans ses Rhipicephalae. Chez les Dermacentor sensu stricto, les dents de l'hypostome sont en file 3/3, parfois 4/4 antérieurement. Chez les Boophilus, les palpes ne sont pas «renflés en dehors, dépassant de beaucoup le bord externe de la base du rostre ", et le rostre n'a pas ses « angles postérieurs de la base prolongés en pointes saillantes et mousses ».

Grâce à l'amabilité de M. le professeur M. André, nous avons pu examiner l'exemplaire type dans les collections du Muséum d'histoire naturelle de Paris. Il s'agit de l'espèce que Schulze (1919) nommera Haemaphysalis otophila.

Neumann a été très certainement induit en erreur sur le genre du fait que l'élargis- 
sement des pa'pes ressemblait à celui de certains Dermacentor, et parce qu'il avait cru y voir des yeux : ce sont vraisemblablement des éclaircissements du bord du scutum. Hormis ce caractère, tout dans le texte original amène à identifier une Haemaphysalis. L'auteur a d'ailleurs omis un détail caractéristique de son exemplaire et de l'espèce en question : il y a seulement neuf festons postérieurs, et non onze comme chez les autres espèces.

Le type de Dermacentor parvus répond entièrement aux descriptions usuelles de Haemaphysalis otophila, selon Pomerancev (1950) ou Feldman-Muhsam (1951). Il n'y a donc pas lieu d'y revenir.

En raison de l'antériorité de la publication de Neumann, Haemaphysalis otophila Schulze, 1919, tombe au rang de synonyme de Haemaphysalis parva (Neumann, 1897) ; de même $H$. otophila schulzei Rondelli 1926.

En 1908, Neumann a décrit une Haemaphysalis parva de Ceylan. Ce terme ne pouvant donc subsister, il doit être remplacé par celui de Haemaphysalis intermedia Warburton et Nuttall, 1908, synonyme de $H$. parva Neumann, 1908, préoccupé (Trapido et Hoogstraal, 1963).

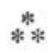

HAEMAPHYSALIS NICOLLEI Larrousse, 1925.

La description de cette espèce, recueillie au Kef (Tunisie) sous une pierre, était trop peu précise pour être interprétée avec certitude. Pourtant Pomerancev (1950) la met en synonymie avec $H$. sulcata (Can. et Fanz., 1877) de même que Feldman-Muhsam (1951).

Cette décision est juste. La femelle existe dans la collection E. Brumpt à l'Institut de Parasitologie de la Faculté de Médecine de Paris. L'exemplaire est à jeun, vraisemblablement de récente mue, car la chitine est claire. Les coxae, par leurs épines et leur pilosité, les trochanters, par leur pilosité, le capitulum, par la morphologie des palpes et des cornes basidorsales, permettent de reconnaître avec certitude $H$. sulcata.

La liste des synonymes est donc la suivante :

Herpetobia sulcata Canestrini et Fanzago, 1877.

Haemaphysalis sulcata (Can. et Fanz.), Neumann, 1897 (espèce condamnée).

Haemaphysalis punctata (Can. et Fanz., 1877) (Neumann, 1897), p.p.

Haemaphysalis cinnabarina punctata (Can. et Fanz.), Nuttall et Warburton, 1915, p.p.

Haemaphysalis nicollei Larrousse, 1925.

Haemaphysalis sulcata sulcata (Can. et Fanz), Schulze, 1925.

Haemaphysalis angorensis Schulze et Schlottke, 1927.

Haemaphysalis cholodkovskyi Olenev, 1928.

Haemaphysalis montana Kamenskii, 1928.

Haemaphysalis cinnabarina punctata musimonis Tonelli-Rondelli, 1930.

Haemaphysalis sulcata (Can. et Fanz.), Pospelova-Strom, 1935.

Haemaphysalis cinnabarina cretica Sénevet et Caminopetros, 1936.

Haemaphysalis beneditoi Gil-Collado, 1938. 
La synonymie avec $H$. sewelli Sharif, 1928, est incertaine.

Haemaphysalis sulcata est une espèce paléarctique qui semble restreinte à la sousrégion méditerranéenne. Sa distribution est la suivante:

Afghanistan : Anastos (1956).

Iran: Delpy (1938), Abbassian-Lintzen (1950); références personnelles: Hessarek, sur mouton, Akinlou, sur lièvre, Abe-Ali, sur tortue.

U.R.S.S. : Pomerancev (1950) ; Anastos (1957).

Irak: Hoogstraal et Kaiser (1959).

Turquie : Schulze et Schlottke (1927: H. angorensis, Ankara); Hoogstraal (1959).

Syrie : Deir ez Zob, chèvre ; Lattaquié, chèvre (Mus. Hist. nat., Paris).

Chypre: Coll. Neumann $\mathrm{n}^{\circ}$ 983, sur Eumeces schneideri, 6 nymphes (Neumann, 1897, 330: H. punctata).

Liban : Joub Yasmina, mouton; Saida, chèvre (Mus. Hist. nat., Paris).

Egypte : Sinaï (Hoogstraal et Kaiser, 1958).

Yemen : Hoogstraal et Kaiser (1959).

Grèce: Mytilène, 1 nymphe sur lézard (coll. Brumpt $=$ Sénevet, 1920: Haemaphysalis sp.).

Bulgarie : Černy (1959).

Roumanie: Feider, Rauchbach et Mironescu (1958).

Albanie : Rosicky, Černy et Luli (1960).

Yougoslavie : Schulze (1927); Mikačic (1952: H. cholodkovskyi).

Italie: Starkoff (1958) ; références personnelles: Gênes (coll. Neumann, $\mathrm{n}^{\circ}$ 973, 3 nymphes sur Vipera aspis $=$ Neumann, 1901, 260: H. punctata).

France: Digne (B. A.) (coll. Neumann $\mathrm{n}^{\circ} 969=$ Neumann, $1897:$ H. punctata).

Espagne : Gil-Collado (1938: Haemaphysalis beneditoi).

Tunisie : Le Kef (Larrousse, 1923 : Haemaphysalis nicollei).

Algérie : Marnia (coll. Neumann $n^{\circ}$ 971: 1 nymphe) ; Sebda (Oran) (coll. Neumann $n^{\circ} 980$ : $1 \sigma^{\circ}$, libre) ; Djebel Ksel (Oran) (coll. Neumann $\mathrm{n}^{\circ} 981,6$ nymphes: Lacerta tingitana); Oran (coll. Neumann $\mathrm{n}^{\circ}$ 982: 1 nymphe: Acanthodactylus vulgaris). Toutes ces références publiées par Neumann, 1901, comme $H$. punctata.

Maroc : Dar Kaïd Mtougui (1 nymphe, Mus. Hist. nat., Paris); Haut Atlas : nn (Psammodromus algirus, Quedenfeltia trachylepharus, Saint-Girons legit).

Canaries: Ténérife (coll. Neumann, $\mathrm{n}^{\circ} 986: 2$ femelles $=$ Neumann, 1901: H. punctata).

Corse : Schulze (1926).

Une remarquable particularité biologique de cette espèce est la prédilection des stades larvaires et nymphaux pour les Reptiles Lacertiliens et Ophidiens.

$$
* *
$$

HAEMAPHYSALIS HIRUDO Neumann, 1897, 341 (non L. Koch, 1877, 786).

Décrite d'une façon assez imprécise sur des exemplaires de Sibérie orientale $(10 \% q$, Amur, Mus. Hamburg), du Japon ( $3 q q$, Japon, Mus. Berlin ; $2 \uparrow q$, Saga, chien, coll. Neumann), de l'Indochine (Saïgon, $2 \%+$, Mus. Paris), cette espèce est renommée par 
son auteur $H$. concinna kochi Neumann, 1905, 238-239, du fait que l'espèce originale de L. Koch lui semble impossible à reconnaître ; cette $H$. concinna kochi serait proche de $H$. concinna C.-L. Koch, 1844 , ce qui justifie son rang de sous-espèce ; ce point de vue est maintenu ultérieurement par son auteur.

Nuttall et Warburton (1915, 456-457) ne modifient pas cette façon de voir, réservant leur jugement du fait de l'absence de mâ'es dans ces lots (qu'ils ont tous examinés) ; « if the corresponding $\delta^{*} \delta^{*}$ should turn out to possess cheliform palps, the attribution to $H$. concinna would be justified, but if this be not the case, we do not think that the similarity of the $q q$ would be sufficient ground to maintain this form as a variety of that species ».

Grâce à l'extrême obligeance de M. le professeur Brizard (Ecole vétérinaire de Toulouse) et de M. le professeur André (Muséum d'histoire naturelle de Paris), nous avons pu revoir certains échantillons observés par Neumann.

Le tube $\mathrm{n}^{\circ} 1366$ de la collection de Toulouse, étiqueté $H$. hirudo, ne contient pas deux femelles, mais une femelle et une nymphe ; sur le registre de la collection, le terme $H$. hirudo est rayé et remplacé par $H$. c. kochi. La femelle correspond à $H$. flava Neumann ( $c f$. Nuttall et Warburton, 1915, 408 ; Keegan et Toshioka, 1957, fig. 21) par les caractères de l'hypostome, des palpes, de la basis capituli et des coxae notamment; il ne s'agit pas de $H$. concinna Koch (comparaison avec des exemplaires de France), ni de H. japonica Warb., 1908 (Pomerancev, 1950: H. japonica douglasi), dont la morphologie est proche de celle des femelles de $H$. flava. La comparaison a été faite avec des femelles de la collection Neumann : $1 \uparrow$ de Saga, cheval (CNm 989), 1 d'Aomori (CNm 987 ; ce lot contient également 1 de $H$. bispinosa Neumann).

La rectification d'identité des spécimens nommés $H$. kochi confirme l'absence de $H$. concinna Koch du Japon, car jusqu'à présent sa présence éventuelle en ce pays ne s'appuyait que sur la synonymie supposée de $H$. concinna kochi avec $H$. concinna.

Les exemplaires de Saïgon ( $2 q q, 1877$, Harmand, Mus. Paris) appartiennent à une autre espèce que $H$.. flava ; la légère saillie externe du $2^{\circ}$ article palpal les rapproche des femelles de Saga, mais les autres caractères les différencient. Leur identité vraisemblable semblerait être $H$. papuana Thorell, 1882 , mais ce fait nécessitera vérification, en raison de la confusion qui règne dans la systématique des Haemaphysalis extrême-orientales, et de la distribution généralement admise pour $H$. papuana, qui correspond à l'Indonésie et la Nouvelle-Guinée. L'essentiel est de savoir leur différence avec les exemplaires de Saga.

Les femelles de l'Amur n'ont pas été réexaminées. En raison de l'état de la systématique des femelles au moment où Neumann et Nuttall ont vu ces lots, nous ne pouvons affirmer ni leur identité entre les tiques de l'Amur et celles de Saga, ni leur différence.

Neumann n'a désigné aucun type, ni pour $H$. hirudo Neum., 1897 , ni pour $H$. $c$. kochi Neum., 1905 ; en 1897 il cite en premier lieu les femelles de l'Amur et, en 1905, les femelles du Japon; c'est d'après leur provenance qu'il avait interprété ces derniers exemplaires comme $H$. hirudo L. Koch; le changement de dénomination de 1905 ne modifie pas le point de vue de Neumann qui accordait aux lots de cette origine une 
signification particulière; seuls de toute la série, les exemplaires de Saga se trouvent dans la collection de Toulouse ; ce dernier fait, joint aux autres, renforce par une considération logique et pratique le choix du lot 1366 comme type de $H$. concinna kochi; cette décision, compte tenu de la synonymie de la femelle type avec $H$. flava, évitera tout changement éventuel dans la nomenclature, dans le cas où les femelles de l'Amur seraient des $H$. japonica Warburton, 1908, où les femelles de Saïgon appartiendraient à une espèce décrite postérieurement à 1905 , si on s'avisait de retenir un de ces derniers lots comme types.

La synonymie de Haemaphysalis flava va donc se présenter de la façon suivante :

*H. flava Neumann, 1897, 333-336 : p.p. ; 1901, 260 : p.p. ; Warburton, 1908, 510 (type : $1 \delta$, Saga, sur herbe, coll. Neumann $\mathrm{n}^{\circ}$ 991) ; H. flava flava Neumann, 1911, 111 : p.p.

$=\quad$ H. flava armata Neumann, 1905, 237-238; 1911, 112.

$=\quad H$. hirudo Neumann, 1897, 341 (non L. Koch, 1877, 786).

$=\quad H$. concinna kochi Neumann, 1905, 238-239; 1911, 111: p.p. (type: 1 \%, Saga, chien, coll. Neumann $\left.\mathrm{n}^{\circ} 1366\right)$.

$=$ ? H. concinna kochi Neumann, 1905, 238-239: p.p. ( $q$ de Sibérie : fleuve Amur).

non $H$. flava Neumann, $1897={ }^{*} H$. campanulata Warburton, 1908 (Neumann, 1897, 335 : Tokio, chien ; coll. Neumann $n^{\circ} 990: 1 \delta^{*} 9 \% q$ chien ; $c f$. Nuttall et Warburton, 1915, 492).

non $H$. flava Neumann, $1897={ }^{*} H$. japonica Warburton, 1908, (Neumann, 1897, 335 : 1 ơ, Miyasaki, cheval).

non $H$. flava Neumann, $1897={ }^{*} H$. intermedia Warburton et Nuttall, 1909, (Neumann, 1897, $336: 4$ ơ ơ, Ceylan ; Mus. Paris ; spécimens vus, P.C.M.).

non $H$. concinna kochi Neumann, $1905=$ ? * H. papuana Thorell, 1882. (Neumann, 1905, 238 : 2 우, Saïgon, Mus. Paris ; rectification P.C.M.).

non $H$. hirudo Neumann, $1897=$ ? * H. papuana Thorell, 1882. (Neumann, 1897, 341 : 2 우, Saïgon, Mus. Paris).

non $H$. hirudo L. Koch, $1877,786=$ nomen incertum.

non $H$. kochi Aragão, 1908, 3-6:1911, 178-181 = H. kohlsi Aragão, 1951, $273=$

* H. juxtakochi Cooley, 1946, 48.

non $* H$. concinna Koch, 1844, 237-238.

(Les bonnes espèces sont indiquées d'une astérisque).

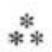

IXODES PUNCTULATUS Canestrini et Fanzago, 1877, p. 183.

Voici la description de l'espèce d'après le texte de Canestrini et Fanzago (1877), repris par Canestrini en 1890 (pp. 502-503) :

« Al ventre osservansi due solchi che si estendono indietro divergendo dall'apertura sessuale fino alla regione anale, e un terzo solco che parte dell'ano e corre fino quasi al contorno posteriore dell'addome... Il contorno posteriore e suddiviso in undici scudetti da 
dodici solchi brevissimi. Il rostro ha, in ciascuna sua metá, tre file longitudinali di denti, i quali diminuiscono di grandezza dall'avanti all'indietro, e sono di forma triangolare col vertice ottuso. I tre denti di ciascuna fila trasversale sono equali di forma e di grandezza. I palpi sono piuttosto brevi. Scudo dorsale... munito di due solchi larghi e quasi paralleli ; contorno pressoche circolare. »

Neumann (1899 p. 160) traduit la description de l'espèce qu'il estime « douteuse en tant qu'Ixodes». Nuttall et Warburton (1911 p. 288) émettent les mêmes réserves: « doubtful if it is an Ixodes ». Starkoff (1958, p. 348) l'estime « indeterminabile ».

Il paraît surprenant que personne n'ait reconnu l'espèce, malgré l'absence d'illustration, car la description est suffisamment explicite.

Puisque Canestrini l'a placée dans les Ixodes, c'est que les palpes sont du type longirostre (quoiqu'il précise : "plutôt courts») et que les yeux sont absents.

Trois caractères par contre interdisent le rapprochement avec les Ixodes:

a) « un troisième sillon part de l'anus et court jusqu'au bord postérieur de l'abdomen »; il s'agit donc d'une disposition metastriata et non prostriata.

b) le contour postérieur de l'abdomen « est divisé en onze écussons par douze sillons très courts »; il ne peut donc s'agir encore d'un prostriata.

c) « le rostre a sur chaque moitié trois files longitudinales de dents, qui diminuent de volume de l'avant vers l'arrière ; les trois dents de chaque file transverses sont égales de forme et de grandeur »; l'hypostome est donc homodonte et non hétérodonte comme chez les Ixodes.

Le choix ne peut donc se porter que sur les genres Dermacentor, Boophilus, Rhipicephalus, Hyalomma et Haemaphysalis. Seul Hyalomma a le rostre long mais présente des yeux. Haemaphysalis n'a pas d'yeux, mais généralement ses palpes sont plus larges que longs. Il existe cependant un sous-genre d'Haemaphysalis : Alloceraea Schulze, dont les palpes sont allongés et simulent ceux d'un Ixodes. L'espèce de Canestrini répond donc à la description de Haemaphysalis inermis Birula, 1895, qui est la seule espèce de ce type en Europe occidentale.

Neumann ainsi que Nuttall et Warburton n'ont pas reconnu Ixodes punctulatus parce que Haemaphysalis inermis était à leur époque une espèce à peine connue dont la présence n'était certaine que dans la région du Caucase.

Ixodes punctulatus Can, et Fanz. (1877), devrait donc avoir la priorité sur Haemaphysalis inermis Birula (1895), s'il n'existait un Ixodes punctulatus Say (1821), synonyme de Dermacentor variabilis (Say, 1821) ; le nom de Canestrini et Fanzago ne peut donc être utililisé et seul demeure valable celui de Birula.

Cette identification ajoute donc l'espèce à la faune italienne. Il était d'ailleurs logique d'y supposer son existence puisqu'elle existe en Yougoslavie et en France, associée le plus souvent à Ixodes ricinus (L.), dans des biotopes à hygrométrie relativement élevée, sous un couvert de chêne rouvre, châtaignier ou hêtre. Canestrini ne donne pas l'origine exacte de son exemplaire mais indique qu'il provient du chevreuil ; il est alors 
vraisemblable qu'une des localités de récolte d'Ixodes ricinus sur cet hôte, par le même auteur, correspond à celle de l'Ixodes punctulatus c'est-à-dire Dosso Tavon (Trentino) ou Treviso (Veneto) (Canestrini, 1890).

Haemaphysalis inermis semble de distribution irrégulière, mais le fait est peut-être dû au hasard ou à la rareté des récoltes en Europe. Voici les grandes lignes de sa distribution :

$$
* *
$$

France: Fontainebleau (Neumann, 1901, p. 262: H. ambigua); Fontainebleau (S.-et-O.), Lancosme (Indre), Chitré (Vienne), sur cerfs (coll. Brumpt) (Morel, 1961) ; Las Illas (P.O.) sur herbe.

Italie: Trentino ou Veneto (Canestrini, 1890).

Yougoslavie : Schulze (1927), Oswald (1938), Mikačic (1949).

Bulgarie: Schulze (1927); Drenski (1955); Černy (1957); coll. Brumpt: Arbanas, Chiflika, Tirnovo, sur bœuf et mouton (envoi de Pavlov).

Tchécoslovaquie: Rosicky (1953); Mačicka (1958).

Roumanie: Feider, Rauchbach et Mironescu (1958).

Iran: Hessarek (Delpy, 1938) ; quelques exemplaires dans la collection Brumpt, recueillis par Delpy, sont des $H$. inermis typiques et non des $H$. aponommoides.

U.R.S.S. : Pomerancev (1950).

\section{Bibliographie}

AbBassian-Lintzen (R.), 1960. - Preliminary list of ticks (Acarina, Ixodoidea) occurring in Iran and their distribution data. Acarologia, 2 (1) : 43-61.

Anastos (G.), 1957. - The ticks, or Ixodidae, of the U.S.S.R. U.S. Dept. Health Educ. Welfare, $\mathrm{n}^{\circ} 528: 398 \mathrm{pp}$.

Birula (A.), 1895. - Ixodidae novi vel parum cogniti Musei Zoologici Academiae Caesareae Scientiarum Petropolitinae. I. Bull. Acad. Imp. Sc. St-Pétesbourg, sér. 5,2 (4) : 353 364.

Canestrini (G.), 1890. - Prospetto dell'acarofauna italiana. IV. Famiglie : Tetranychini, Ixodini, Argasini.. Padova (éd. Prosperini), 4: 427-540.

—et Fanzago (F.), 1877. - Intorno agli Acari italiani. Atti R. Ist. veneto Sc. Lett. Arti, 4 (1) : 69-208.

ČernY (V.), 1959. - Ein Beitrag zur Zeckenfauna Bulgariens. Brnenske Zakladny Ceskosl. Akad. Vēt., 31 (392 (7)) : 361-364.

DelPy (L. P.), 1938. - Les espèces iraniennes du genre Haemaphysalis Koch, 1894. Identité d'Haemaphysalis cholodkovskyi Olenev, 1928, et H. cinnabarina cretica Senevet et Caminopetros, 1936. Ann. Parasit. hum. comp., 16 (1) : 1-10.

DrensKI (P.), 1955. - Sistav i rasprostanenie na kirlešite v Bulgaria. Bul. Inst. Zool., Acad. St. Bulg., 4-5: 109-160.

Feider (Z.), Rauchbach (C.) et Mironescu (I.), 1958. - Die Zecken der rumänischen Volksrepublik. Ceskoslov. Parasit., 5 (2) : 71-87. 
Feldman-Musham (B.), 1951. - A note on east-mediterranean species of the genus Haemaphysalis. Bull. Res. Coun. Israel. 1 (3): 96-107.

Gil Collado (J.), 1938. — Los acaros de España (Ixodoideos). Ed. Brotéria, 7 (3) : 99-109.

Hoogstraal (H.), 1959. - Biological observations on certain turkish Haemaphysalis ticks (Ixodoidea, Ixodidae). J. Parasit., 45 (2) : 227-232.

- et Kaiser (M.), 1958. - The ticks (Ixodoidea) of Egypt. A brief review and keys. J. egypt. publ. Health Assoc., 33 (3) : 51-85.

- - 1958. - The ticks (Ixodoidea) of Iraq : keys, hosts and distribution. J. iraqi. med. Profession., 6 (2-3) : 1-22.

- - 1959. - Ticks (Ixodoidea) of Arabia, with special reference to the Yemen. Fieldiana, Zoology, 39 (28) : 297-322.

KAMENSKII (S. V.), 1928. - Tri goda rabot po piroplazmozam i im podobnhym zabolevaniiam domašnix jivotnhyx, Severnogo Kavkaza, 1924, VI, 1927. I. Klešči domašnix jivotnhyx S.K. II. Piroplasmidae domašnix jivotnhyx S.K. III. Opredelitel kleščei domjivotnhyx S.K. (Three years'work on piroplasmosis and on similar diseases of domestic animals in North Caucasus. I. Ticks of domestic animals. II. Piroplasmidae of domestic animals. III. Key to the ticks of domestic animals). Rostov-na-Donu, $31 \mathrm{pD}$.

LARRousse (F. L.), 1925. - Nouvelle espèce tunisienne du genre Haemaphysalis: H. nicollei n. sp. Arch. Inst. Pasteur Tunis, 14 (1): 97-100.

MačrčKa (O.), 1958. - K bionomii Haemaphysalis inermis Birula u nas. Ceskosl. Parasit., 5 : 121-124.

MIKačrc (D.), 1949. - Krpeiji (Ixodidae) Cresa i Lošinja s orsvtom na geografsku proširenost pojedinih vrsta krpelja u Jugoslaviji. (Ixodidés des îles de Cres et Losin, avec une carte de distribution des espèces de tiques en Yougoslavie), Vet. Archiv. Zagreb, 19 (1-2) : 14-32.

—, 1952. - Les piroplasmoses en Yougoslavie. Bull. Off. intern. Epiz., 1962, 38 : 575-592 pp.

Morel (P. C.) 1961. - Ixodidés (incl. Argasidés). Trentenaire de la Station expérimentale de Parasitologie de Richelieu (I.-et-L.). Ann. Parasit. hum. comp., 36 (3) : 337-340.

Neumann (G.), 1897. - Révision de la famille des Ixodidés. II. Mém. Soc. Zool. France, 10 (3-4) : 324-420.

—, 1901. — Révision de la famille des Ixodidés. IV. Ibid., 14 (2-3) : 249-372.

—, 1908. - Notes sur les Ixodidés. VI. Arch. Parasit. Paris, 12 (1) : 5-27.

—, 1911. - Ixodidae. Das Tierreich, 26: 1-169.

Nuttall (G. H. F.) et Warburton (C.), 1911. - The genus Ixodes (in Nuttall et coll., ticks. A monograph of the Ixodoidea II). Cambridge, Univ. Press. 1-19, 105-348.

- - 1915. - The genus Haemaphysalis (in Nuttall et coll., ticks. A monograph of the Ixodoidea III). Cambridge, Univ. Press., 349-550.

Olenev (N. O.), 1928. - K sistematike i geografičeskomu rasprostraneniu o kleščei Ixodoidea. (Sur la classification et la distribution géographique des Ixodidés). Dokl, Akad. Nauk S.S.R., n 2 : 29-34 : R.A.E., B, 1928, 15 (9) : 184.

Pomerancev (B. I.), 1950. - Fauna S.S.S.R. Paukoobraznhye, 4. Iksodovhye klešči. Izd. Akad. Nauk S.S.S.R., Moskva-Leningrad, 4 (2) : 224 pp. Fauna of the U.S.S.R. Arachnida, 
4. Ixodid ticks. Edit. Acad. Sci. U.S.S.R., Moskva-Leningrad, 4 (2) : 199 pp.; English translation by H. Elbl, édit. G. Anastos, Amer. Inst. biol. Sci., Washington, 6, D.C. and New-York, 2000 P. street.

Pospelova-Strom (M. V.), 1935. - Zur Systematik der Ixoden Gattung Haemaphysalis Koch. Trav. Fil. Acad. Sci. U.R.S.S. Tad., 5 : 205-217.

Rondelli (M.), 1926. - Missione zoologica del $\mathrm{D}^{\mathrm{r}}$ E. Festa in Cirenaica. Ixodoidea. Res. biol., 1 (4) : 45-48.

RosickY (B.), 1953. - Bionomiko-faunisticky nastin klištat (Ixodidae) z Uzemi C.S.R. Zool. Ent. Listy, 2 : 120-130.

SEnEvet (G.), 1920. - Note sur quelques Ixodes parasites des animaux domestiques recueillis à Mytilène de février à juin 1916. Bull. Soc. Path. Exot., 13 (4) : 260-261.

Schulze (P.), 1919. - Ein Beitrag zur Zecken fauna Mazedoniens. Sitz.-Ber. Ges. Naturf. Fr. Berlin, 1918, 1-2:61-66.

—, 1927. - Beitrag zur Kenntniss der Zecken Europas. Ibid., 1925, n 1-10: 109-126.

—, 1926. - Ixodoidea. Beiträge zur Kenntniss der Land = und Süsswasserfauna Korsikas. I. Ergebnisse der $\mathrm{D}^{\mathrm{r}}$ Paul Schottländer Lehrexpedition des Jahres 1914. Mitt. Zool. Mus. Berlin, 12 (2) : 273.

— et Schlotrke (E.), 1927. - Eine neue Haemaphysalis Art aus Angora (Ixodidae). Zool. Anz., 74 (7-10): 157-159.

Trapido (H.) et Hoogstraal (H.), 1963. - Status of Haemaphysalis bispinosa var. intermedia Warburton et Nuttall, 1909, next available name for H. parva Neumann, 1908 (preoccupied) (Ixodoidea, Ixodidae). J. Parasit., 49 (4) : 691-692.

Institut d'Elevage et de Médecine vétérinaire des Pays tropicaux, Paris Laboratoire national des Recherches vétérinaires, Dakar 